\title{
Use of second-generation antipsychotics in the acute inpatient management of schizophrenia in the Middle East
}

This article was published in the following Dove Press journal:

Neuropsychiatric Disease and Treatment

I April 2015

Number of times this article has been viewed

\section{Sulaiman Alkhadhari' \\ Nasser Al Zain ${ }^{2}$ \\ Tarek Darwish ${ }^{3}$ \\ Suhail Khan ${ }^{4}$ \\ Tarek Okasha ${ }^{5}$ \\ Hisham Ramy ${ }^{5}$ \\ Talaat Matar Tadros ${ }^{6}$}

'Kuwait Center for Mental Health, Safat, Kuwait; ${ }^{2} \mathrm{Al}$ Amal Complex for Mental Health Hospital, Dammam, Saudi Arabia; ${ }^{3}$ Behavioural Science Pavilion, Sheikh Khalifa Medical City, Abu Dhabi, United Arab Emirates; ${ }^{4}$ Jeddah Psychiatric Hospital, Jeddah, Saudi Arabia; ${ }^{5}$ Institute of Psychiatry, Ain Shams University, Cairo, Egypt; ${ }^{6}$ lbrahim Bin Hamad Obaidallah and Seif Bin Ghubash Hospitals, Ras Alkhaimah, United Arab Emirates
Correspondence: Hisham Ramy Institute of Psychiatry, Ain Shams University, 37 Fareed Street, Heliopolis, Cairo, Egypt Tel +201271010846

Email hisham.ramy67@gmail.com
Background: Management of acute psychotic episodes in schizophrenic patients remains a significant challenge for clinicians. Despite treatment guidelines recommending that secondgeneration antipsychotics (SGAs) should be used as monotherapy, first-generation antipsychotics, polypharmacy, and lower than recommended doses are frequently administered in clinical practice. Minimal data exist regarding the use of SGAs in the Middle East. The objective of this study was to examine the discrepancies between current clinical practice and guideline recommendations in the region.

Methods: RECONNECT-S Beta was a multicenter, noninterventional study conducted in Egypt, Kuwait, Saudi Arabia, and the United Arab Emirates to observe the management of schizophrenic patients who were hospitalized due to an acute psychotic episode. Patients underwent one visit on the day of discharge. Demographic and medical history, together with data on antipsychotic treatment and concomitant medication during the hospitalization period and medication recommendations at discharge were recorded.

Results: Of the 1,057 patients, $180(17.0 \%)$ and 692 (65.5\%) received SGAs as monotherapy and in combination therapy, respectively. Overall, the most frequently administered medications were given orally, and included risperidone (40.3\%), olanzapine $(32.5 \%)$, and quetiapine (24.6\%); the doses administered varied between countries and deviated from the recommended guidelines. Upon discharge, $93.9 \%$ of patients were prescribed SGAs as maintenance therapy, and $84.8 \%$ were prescribed the same medication(s) as during hospitalization.

Conclusion: Current clinical practice in the Middle East differs from guideline recommendations. Patients frequently received antipsychotics in combination therapy, by various methods of administration, and at doses above and below the recommended guidelines for the management of their acute psychotic episodes.

Keywords: schizophrenia, Middle East, second-generation antipsychotic, acute psychotic episode, treatment guidelines

\section{Introduction}

Schizophrenia is a severe form of mental illness presenting with long duration, bizarre delusions, nonaffective psychosis, and negative symptoms, ${ }^{1}$ and is estimated by the World Health Organization to affect approximately 24 million people worldwide. ${ }^{2}$ The disease burden in the Middle East is markedly greater than in developed countries, ${ }^{3}$ with age-standardized disability-adjusted life years attributable to schizophrenia in 2004 estimated to be 273, 270, 269, and 267 per 100,000 inhabitants in Egypt, Saudi Arabia, Kuwait, and the United Arab Emirates, respectively, compared with 164 per 100,000 inhabitants in Australia. ${ }^{4}$ 
Acute psychotic episodes in patients with schizophrenia remain a significant challenge for clinicians; ${ }^{5}$ approximately $40 \%$ of all schizophrenic individuals are poorly adherent to their antipsychotics at any given time, and often suffer from exacerbations and relapses that require hospitalization. ${ }^{6}$ There is a paucity of information regarding the use of antipsychotics for the agitation component during these acute episodes in the Middle East. In Egypt, electroconvulsive therapy is used according to Egyptian Mental Health Act regulations and Mental Health Secretariat of the Ministry of Health guidelines to reduce patient hospital stay and emotional turmoil, improve response to antipsychotic medications, and lower the dosage of antipsychotics administered. ${ }^{7,8}$ No specific treatment guidelines for schizophrenia exist in Saudi Arabia or in the United Arab Emirates. However, both countries follow guidelines published by the American Psychiatric Association, ${ }^{9}$ and also consult the UK minimum recommended and maximum licensed doses of antipsychotics published in The Maudsley Prescribing Guidelines in Psychiatry..$^{10}$ The American Psychiatric Association guidelines recommend that selection of an antipsychotic agent be guided by the patient's past medication history, with preference for a specific medication based on past experience, current symptoms, co-occurring conditions, and other concurrent treatments. Monotherapy with second-generation agents should be considered a first-line option for patients in the acute phase, mainly because of the decreased risk of extrapyramidal side effects and tardive dyskinesia accompanying the first-generation antipsychotics (FGAs). ${ }^{11,12}$

Despite treatment guidelines usually indicating that second-generation antipsychotics (SGAs) are preferable and should be used in monotherapy, available evidence has revealed frequent use of FGAs, polypharmacy, intramuscular route of administration, and use of SGAs at lower than recommended doses. ${ }^{13}$ This suggests that in addition to guidelines, physicians should also use their discretion and experience to determine the most appropriate treatments for their patients. The aim of the RECONNECT-S Beta study was to describe the real-life use of SGAs in the acute inpatient management of schizophrenia in a noninterventional setting, in order to gain an understanding of disparities that may exist between current clinical practice and guideline recommendations followed in the Middle East.

\section{Materials and methods Study design}

This was a noninterventional, multicenter study, registered as NCT01544608, to observe the management of patients with schizophrenia hospitalized due to an acute psychotic episode in Egypt, Kuwait, Saudi Arabia, and the United Arab Emirates. All psychiatrists recruited into the study (full details included in Table S1) had at least 10-15 years' experience, represented different geographical locations, and were responsible for the inpatient wards that subjects participating in the study had stayed on. Public, private, general, and mental health hospitals were included in the study. Subjects participated in one visit on their day of discharge after hospitalization due to an acute psychotic episode (Figure 1). At the study visit, data on demographics, diagnosis, and medical history were recorded. Data on antipsychotic treatments and concomitant medications were collected for the hospitalization period, along with the recommended treatment regimen at discharge. The first subject's first visit was on April 7, 2012, and the last subject's last visit was on December 31, 2012.

\section{Patient enrolment criteria}

Patients were eligible for enrolment in the study if they were $\geq 18$ years, met the diagnostic criteria for schizophrenia stated in the Diagnostic and Statistical Manual of Mental Disorders, Fourth Edition, and were hospitalized due to an acute psychotic episode. The study required that the subject had the ability to understand and comply with the requirements of the study, as judged by the investigator. Written informed consent was obtained from the subject and/or his/ her legal representative (as per local regulatory requirements).
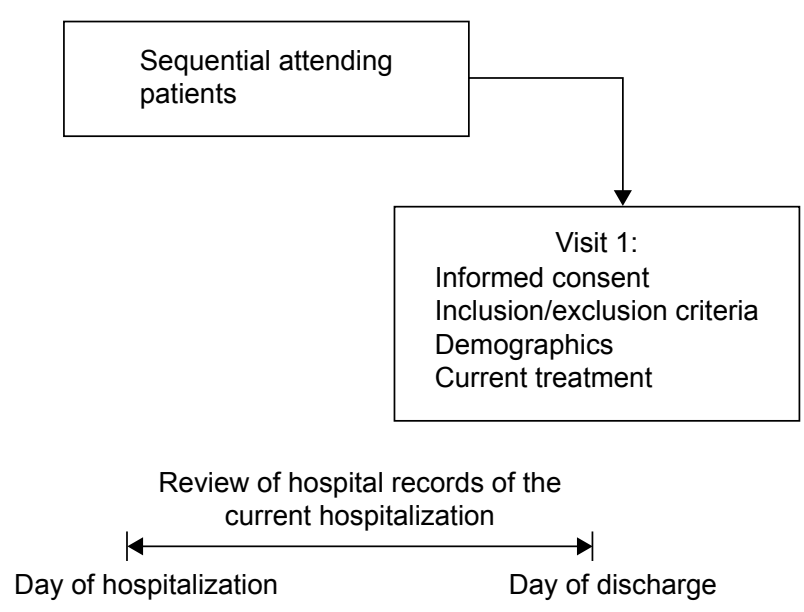

Figure I Study design.

Notes: Patients made one visit on the day of their discharge after hospitalization due to an acute psychotic episode; during this visit, data on demographics, diagnosis, and medical history were recorded. In addition, data on antipsychotic treatment and concomitant medication were collected for the hospitalization period, together with the recommendation at discharge. Any patients who met the enrolment criteria ( $\geq 18$ years and met the diagnostic criteria for schizophrenia stated in Diagnostic and Statistical Manual of Mental Disorders, Fourth Edition) and had signed a written informed consent form were included in the study. 
Any patients who both met the enrolment criteria upon discharge from hospital and had signed the written informed consent form were included in the study; thus, the study sample was not randomized. Exclusion criteria were current participation in a clinical trial or previous enrolment in the current study (in the case of rehospitalization).

\section{Study objectives}

The primary objective of the study was to describe the use of SGAs in patients with schizophrenia during hospitalization due to an acute psychotic episode, by evaluation of drug, dose, and mode of administration of the medication. Duration of administration was noted by length of inpatient stay. Secondary objectives were to describe: use of SGAs as monotherapy (ie, the sole medication administered to patients) during the hospitalization period; use of combinations of antipsychotics during the hospitalization period; the main criteria used for selection of an antipsychotic to treat acute episodes of schizophrenia; use of psychometric rating scales to evaluate disease symptoms during the hospitalization period; use of concomitant psychiatric medication (other than SGAs) during hospitalization; and the correlation between antipsychotic medication used during hospitalization and maintenance therapy recommended upon discharge.

\section{Safety}

No proactive collection of safety data was performed during this study owing to its noninterventional nature. Only spontaneously mentioned adverse events were reported as required by the post-marketing pharmacovigilance regulations.

\section{Statistical analyses}

A descriptive analysis approach (including frequency tables) was used because of the noninterventional design of the study. As appropriate, a two-sided $95 \%$ confidence interval was obtained for the population estimation of the variables. All calculations and summaries were produced using SAS version 9.2 (SAS Institute, Cary, NC, USA), with medications coded using the WHO Drug Dictionary, version 12.1.

\section{Ethics}

The study was performed in accordance with the ethical principles laid down in the Declaration of Helsinki, International Conference on Harmonisation Good Clinical Practice, and the applicable legislation. The investigators performed this noninterventional study in accordance with the regulations and guidelines governing medical practice and ethics in their country.

\section{Results Study participants}

Forty-four investigators participated in the study, comprising 29 in Egypt, ten in Saudi Arabia, five in the United Arab Emirates, and one in Kuwait (data from Kuwait and the United Arab Emirates are combined and referred to as "Gulf States" in this study). A total of 1,076 study patients were recruited by investigators, of whom 1,057 met the eligibility criteria (480 in Egypt, 333 in Saudi Arabia, and 244 in the Gulf States, Table 1).

\section{Patient demographics}

Patient demographics are shown in Table 2. All analyses were carried out on the per protocol population; in this study, no treatment recommendations were made, so use of this term merely signifies those patients who met all of the inclusion criteria and none of the exclusion criteria, and for whom data were available. The per protocol population comprised 1,057 patients with a mean age of $34.4 \pm 11.44$ years, of whom $792(74.9 \%)$ were male. The majority of participants had no history of harmful alcohol or drug use $(95.6 \%$ and $85.7 \%$, respectively). Approximately half of the study patients reported psychosocial problems, with $8.5 \%$ having made at least one suicide attempt in the previous 2 years.

Overall, $19.3 \%$ of patients had concurrent, secondary psychiatric conditions, in addition to their primary diagnosis of schizophrenia, and $18.8 \%$ had other general medical conditions (Table 3). Within the month prior to hospitalization, only $45.8 \%$ of patients overall had used antipsychotic medications, and of these, risperidone (41.9\%) and olanzapine

Table I Study population (n)

\begin{tabular}{lllll}
\hline & Egypt & Saudi Arabia & Gulf States & Overall \\
\hline Enrolled & 480 & 351 & 245 & 1,076 \\
Per protocol population & 480 & 333 & 244 & 1,057 \\
Did not meet all inclusion criteria or & 0 & 18 & 1 & 19
\end{tabular}

met at least one exclusion criterion

Notes: Gulf States, represents combined data from Kuwait and the United Arab Emirates. All analyses were carried out on the per protocol population; in this study, no treatment recommendations were made, so the use of this term merely signifies those patients who met all of the inclusion criteria and none of the exclusion criteria, and for whom data are available. 
Table 2 Patient demographics

\begin{tabular}{|c|c|c|c|c|}
\hline & $\begin{array}{l}\text { Egypt } \\
(n=480) \text { n (\%) }\end{array}$ & $\begin{array}{l}\text { Saudi Arabia } \\
(n=333) n(\%)\end{array}$ & $\begin{array}{l}\text { Gulf States } \\
(n=244) n(\%)\end{array}$ & $\begin{array}{l}\text { Overall } \\
(n=I, 057) n(\%)\end{array}$ \\
\hline \multicolumn{5}{|l|}{ Sex } \\
\hline Male & 37I (77.3) & $267(80.2)$ & I54 (63.I) & 792 (74.9) \\
\hline Female & $109(22.7)$ & $65(19.5)$ & $88(36.1)$ & $262(24.8)$ \\
\hline \multicolumn{5}{|l|}{ Age (years) } \\
\hline Responses & 476 & 288 & 238 & 1,002 \\
\hline Mean & 32.2 & 36.8 & 36.2 & 34.4 \\
\hline SD & 11.44 & 10.90 & 11.25 & 11.44 \\
\hline \multicolumn{5}{|l|}{ Total years of education } \\
\hline Responses & 450 & 275 & 178 & 903 \\
\hline Mean & 12.5 & 9.9 & 10.0 & 11.2 \\
\hline SD & 4.53 & 4.42 & 4.19 & 4.60 \\
\hline \multicolumn{5}{|l|}{ Current employment status } \\
\hline Unemployed & $230(47.9)$ & $218(65.5)$ & I54 (63.I) & $602(57.0)$ \\
\hline Employed & $101(21.0)$ & $49(14.7)$ & $45(18.4)$ & $195(18.4)$ \\
\hline Self-employed & $51(10.6)$ & $8(2.4)$ & $4(1.6)$ & $63(6.0)$ \\
\hline Student & $32(6.7)$ & I5 (4.5) & $4(1.6)$ & $5 I(4.8)$ \\
\hline Sickness pension & $2(0.4)$ & $2(0.6)$ & $33(13.5)$ & $37(3.5)$ \\
\hline Other & $44(9.2)$ & $5(1.5)$ & 0 & $49(4.6)$ \\
\hline Unknown & $4(0.8)$ & $3(0.9)$ & 0 & $7(0.7)$ \\
\hline Missing & $\mathrm{I}(0.2)$ & 0 & 0 & $\mathrm{I}(<0 . \mathrm{I})$ \\
\hline \multicolumn{5}{|l|}{ Children $<18$ years in household } \\
\hline No & $355(74.0)$ & $222(66.7)$ & $150(6 \mid .5)$ & $727(68.8)$ \\
\hline Yes & $119(24.8)$ & $85(25.5)$ & $72(29.5)$ & $276(26.1)$ \\
\hline Unknown & $3(0.6)$ & $21(6.3)$ & $6(2.5)$ & $30(2.8)$ \\
\hline Missing & $3(0.6)$ & $5(1.5)$ & $16(6.6)$ & $24(2.3)$ \\
\hline \multicolumn{5}{|l|}{ Current marital status } \\
\hline Single & $326(67.9)$ & $211(63.4)$ & $142(58.2)$ & $679(64.2)$ \\
\hline Married/partner & $106(22.1)$ & $73(21.9)$ & $67(27.5)$ & $246(23.3)$ \\
\hline Divorced/separated & $39(8.1)$ & $48(14.4)$ & 34 (13.9) & $121(11.4)$ \\
\hline Widow/widower & $7(1.5)$ & I $(0.3)$ & I $(0.4)$ & $9(0.9)$ \\
\hline Unknown & $\mathrm{I}(0.2)$ & 0 & 0 & $\mathrm{I}(<0 . \mathrm{I})$ \\
\hline Missing & $\mathrm{I}(0.2)$ & 0 & 0 & $\mathrm{I}(<0 . \mathrm{I})$ \\
\hline \multicolumn{5}{|l|}{ Living situation } \\
\hline Living with parent(s) & $299(62.3)$ & $189(56.8)$ & $135(55.3)$ & $623(58.9)$ \\
\hline Living with husband/wife/partner & $104(21.7)$ & $68(20.4)$ & $57(23.4)$ & $229(21.7)$ \\
\hline Living alone & $38(7.9)$ & $15(4.5)$ & $9(3.7)$ & $62(5.9)$ \\
\hline Living with other adult & $34(7.1)$ & $41(12.3)$ & $38(15.6)$ & $113(10.7)$ \\
\hline Other & $4(0.8)$ & $14(4.2)$ & $4(1.6)$ & $22(2.1)$ \\
\hline Unknown & $\mathrm{I}(0.2)$ & $6(1.8)$ & 0 & $7(0.7)$ \\
\hline Missing & 0 & 0 & I $(0.4)$ & $\mathrm{I}(<0 . \mathrm{I})$ \\
\hline \multicolumn{5}{|l|}{ Harmful alcohol use } \\
\hline No & $458(95.4)$ & $321(96.4)$ & $232(95.1)$ & I,0II (95.6) \\
\hline Yes & $20(4.2)$ & $10(3.0)$ & II (4.5) & $41(3.9)$ \\
\hline Unknown & $2(0.4)$ & $2(0.6)$ & $\mathrm{I}(0.4)$ & $5(0.5)$ \\
\hline \multicolumn{5}{|l|}{ Harmful drug use } \\
\hline No & $383(79.8)$ & $289(86.8)$ & $234(95.9)$ & $906(85.7)$ \\
\hline Yes & $97(20.2)$ & $42(12.6)$ & $8(3.3)$ & 147 (I3.9) \\
\hline Unknown & 0 & $2(0.6)$ & $\mathrm{I}(0.4)$ & $3(0.3)$ \\
\hline Missing & 0 & 0 & $\mathrm{I}(0.4)$ & $\mathrm{I}(<0 . \mathrm{I})$ \\
\hline \multicolumn{5}{|l|}{$\begin{array}{l}\text { Number of suicide attempts during } \\
\text { previous years }\end{array}$} \\
\hline 0 & $416(86.7)$ & $314(94.3)$ & $225(92.2)$ & $955(90.4)$ \\
\hline $\mathrm{I}-2$ & $4 \mid(8.5)$ & $14(4.2)$ & $15(6.1)$ & $70(6.6)$ \\
\hline 3 or more & $12(2.5)$ & $4(1.2)$ & $4(1.6)$ & $20(1.9)$ \\
\hline Unknown & II (2.3) & $\mathrm{I}(0.3)$ & 0 & $12(1.1)$ \\
\hline
\end{tabular}


Table 2 (Continued)

\begin{tabular}{lllll}
\hline & $\begin{array}{l}\text { Egypt } \\
(\mathbf{n}=\mathbf{4 8 0}) \mathbf{n}(\%)\end{array}$ & $\begin{array}{l}\text { Saudi Arabia } \\
(\mathbf{n}=\mathbf{3 3 3}) \mathbf{n}(\mathbf{\%})\end{array}$ & $\begin{array}{l}\text { Gulf States } \\
(\mathbf{n}=\mathbf{2 4 4}) \mathbf{n}(\%)\end{array}$ & $\begin{array}{l}\text { Overall } \\
(\mathbf{n}=\mathbf{I}, \mathbf{0 5 7}) \mathbf{n}(\%)\end{array}$ \\
\hline Psychosocial problems & & & & \\
No & $233(48.5)$ & $173(52.0)$ & $108(44.3)$ & $514(48.6)$ \\
Yes & $242(50.4)$ & $157(47.1)$ & $132(54.1)$ & $531(50.2)$ \\
Unknown & $5(1.0)$ & $3(0.9)$ & $4(1.6)$ & $12(1.1)$ \\
\hline
\end{tabular}

Note: Gulf States, represents combined data from Kuwait and the United Arab Emirates.

Abbreviation: SD, standard deviation.

(24.6\%) were the most frequently used. While the incidence of concurrent psychiatric conditions was greatest in Egypt, the occurrence of other general medical conditions and prior antipsychotic use was lowest.

\section{Primary objectives}

\section{Length of inpatient stay}

Mean patient length of hospital stay varied between countries; the shortest stays were observed in Egypt, followed by the Gulf States and Saudi Arabia (27.0, 34.0, and 41.5 days, respectively, Table 4).

\section{Use of SGAs}

The choice of medication administered during hospitalization was at the discretion of the consulting physician. The majority of patients $(1,011 / 1,057,95.6 \%)$ were treated with at least one SGA (Table 5). Overall, the most frequently used SGAs were risperidone (40.3\%), olanzapine $(32.5 \%)$, and quetiapine (24.6\%). However, variations were observed in antipsychotic administration per country: risperidone was most commonly administered in Egypt (211/480, 44.0\%), while olanzapine was more commonly used in Saudi Arabia (138/333, 41.4\%) and the Gulf States $(103 / 244,42.2 \%)$. Nearly all patients $(1,019 / 1,057,96.4 \%)$ were given oral medication, with only nine and 29 patients, respectively, receiving olanzapine and risperidone via the intramuscular route. Of note, a greater percentage of participants (13.1\%) in the Gulf States received treatment for psychiatric symptoms with a medication categorized as a nonantipsychotic compared with Egypt $(2.5 \%)$ or Saudi Arabia (2.1\%).

\section{Doses of SGAs administered}

Variation was observed between the doses of SGAs most commonly administered in different countries (Table 5). The median orally administered doses of risperidone, olanzapine, and quetiapine were highest in Egypt, whereas the median orally administered dose of clozapine was lowest in this country.

\section{Secondary objectives}

\section{Use of monotherapy or combination therapy}

Although the majority of patients $(1,011,95.6 \%)$ received at least one SGA during hospitalization, only 180/1,057 patients (17.0\%) received SGAs as monotherapy, whilst 139/1,057 patients (13.2\%) received more than one SGA (Table 6). The majority of patients $(692,65.5 \%)$ were treated with SGAs in combination with other psychiatric medications.

Table 3 Medical history of patients

\begin{tabular}{|c|c|c|c|c|}
\hline & Egypt $(n=480)$ & Saudi Arabia $(n=333)$ & Gulf States $(n=244)$ & Overall $(n=1,057)$ \\
\hline \multicolumn{5}{|c|}{ Concurrent secondary psychiatric conditions, n (\%) } \\
\hline No & $36 \mathrm{I}(75.2)$ & $279(83.8)$ & $210(86.1)$ & $850(80.4)$ \\
\hline Yes & $117(24.4)$ & $53(15.9)$ & $34(13.9)$ & $204(19.3)^{\mathrm{a}}$ \\
\hline Unknown & $2(0.4)$ & I $(0.3)$ & 0 & $3(0.3)$ \\
\hline \multicolumn{5}{|l|}{ General medical conditions, n (\%) } \\
\hline No & $402(83.8)$ & $259(77.8)$ & I88 (77.0) & $849(80.3)$ \\
\hline Yes & $76(15.8)$ & $72(21.6)$ & $51(20.9)$ & $199(18.8)$ \\
\hline Unknown & I $(0.2)$ & I $(0.3)$ & 0 & $2(0.2)$ \\
\hline Missing & I (0.2) & $\mathrm{I}(0.3)$ & $5(2.0)$ & $7(0.7)$ \\
\hline $\begin{array}{l}\text { Treatment with at least one antipsychotic } \\
\text { medication, } n(\%)\end{array}$ & $146(30.4)$ & $186(55.9)$ & $152(62.3)$ & $484(45.8)$ \\
\hline
\end{tabular}

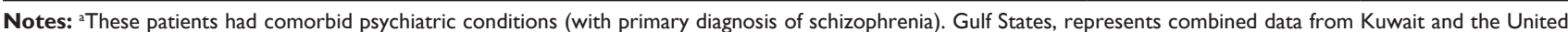
Arab Emirates. 
Table 4 Length of hospital stay (days)

\begin{tabular}{lllll}
\hline & Egypt $(\mathbf{n}=\mathbf{4 7 9})$ & Saudi Arabia $(\mathbf{n}=\mathbf{2 9 3})$ & Gulf States $(\mathbf{n}=\mathbf{2 2 6})$ & Overall $(\mathbf{n}=\mathbf{9 9 8})$ \\
\hline Mean & 27.0 & 41.5 & 34.0 & 32.8 \\
SD & 21.9 & 42.8 & 32.6 & 32.3 \\
Minimum & 2.0 & 3.0 & 2.0 & 2.0 \\
Maximum & 169.0 & 191.0 & 171.0 & 191.0 \\
$95 \%$ Cl* & $(25.0-29.0)$ & $(36.5-46.4)$ & $(29.7-38.3)$ & $(30.8-34.8)$ \\
\hline
\end{tabular}

Notes: Subjects with hospital duration $>200$ days were considered outliers and are not included in the summary statistics for hospital duration. Gulf States, represents combined data from Kuwait and the United Arab Emirates. *Normal approximation for the mean.

Abbreviations: $\mathrm{Cl}$, confidence interval; $\mathrm{SD}$, standard deviation.

Overall, 458 (43.3\%) patients received at least one concomitant non-SGA(s), and administration was significantly more common in Egypt $(274,57.1 \%)$ than in Saudi Arabia $(129,38.7 \%)$ or the Gulf States $(55,22.5 \%)$. In addition, $116(11.0 \%), 110(10.4 \%), 98$ (9.3\%), and 78 (7.4\%) patients were treated with antiepileptics, antidepressants, anxiolytics, and anticholinergic agents, respectively. Electroconvulsive therapy and psychological treatment (including psychotherapy [individual or group sessions], psychoeducation, and family education) was also given to $33.7 \%$ and $39.5 \%$ of patients, respectively; marked differences in use of these modalities were observed between countries. Electroconvulsive therapy was given to $56.9 \%, 20.7 \%$, and $5.7 \%$ of patients in Egypt, Saudi Arabia and the Gulf States, respectively; psychological treatment was given to $56.5 \%, 14.4 \%$, and $40.2 \%$ of patients in Egypt, Saudi Arabia, and the Gulf States, respectively.

\section{Criteria used for selection of an antipsychotic}

The most frequently stated rationales for selection of the SGA medication chosen were current symptoms (62.2\%), medication history (26.5\%), or preference based on physician's personal experience $(20.4 \%)$, with physicians most commonly citing current symptoms in Egypt and Saudi Arabia (80.0\% and $61.0 \%$, respectively), and preference based on personal clinical experience in the Gulf States (47.1\%).

\section{Use of psychometric scales to evaluate patients' symptoms}

The majority of the study patients (94.8\%) had their treatment evaluated in accordance with the clinical experience of the consulting physician; psychometric rating scales were only used to evaluate the response of symptoms of the disease to treatment during hospitalization for $0.8 \%$ of patients, with no reported use in the Gulf States.

Table 5 Median daily doses of the most commonly administered antipsychotic drugs

\begin{tabular}{|c|c|c|c|c|}
\hline & Egypt $(n=480)$ & Saudi Arabia $(n=333)$ & Gulf States $(n=244)$ & Overall $(n=I, 057)$ \\
\hline \multicolumn{5}{|l|}{ Clozapine (mg/day) } \\
\hline Oral route, $\mathrm{n}(\%)$ & 155 (33.9) & $20(6.3)$ & $17(7.5)$ & $192(19.1)$ \\
\hline Median & 150.0 & 300.0 & 300.0 & 200.0 \\
\hline \multicolumn{5}{|c|}{ UK licensed maximum dose: $900 \mathrm{mg} / \mathrm{day}$} \\
\hline \multicolumn{5}{|c|}{ Olanzapine (mg/day) } \\
\hline Oral route, $\mathrm{n}(\%)$ & $97(21.2)$ & $134(42.1)$ & $103(45.2)$ & $334(33.3)$ \\
\hline Median & 20.0 & 17.5 & 15.0 & 20.0 \\
\hline IM route, $\mathrm{n}(\%)$ & $5(1.1)$ & $4(1.3)$ & 0 & $9(0.9)$ \\
\hline Median & 20.0 & 20.0 & - & 20.0 \\
\hline \multicolumn{5}{|c|}{ UK licensed maximum dose: 20 mg/day } \\
\hline \multicolumn{5}{|l|}{ Quetiapine (mg/day) } \\
\hline Oral route, $\mathrm{n}(\%)$ & $119(26.0)$ & $88(27.7)$ & $53(23.2)$ & $260(25.9)$ \\
\hline Median & 500.0 & 450.0 & 300.0 & 400.0 \\
\hline \multicolumn{5}{|c|}{ UK licensed maximum dose: $750-800 \mathrm{mg} /$ day } \\
\hline \multicolumn{5}{|c|}{ Risperidone (mg/day) } \\
\hline Oral route, n (\%) & $208(45.5)$ & 117 (36.8) & $72(31.6)$ & $397(39.6)$ \\
\hline Median & 6.0 & 4.0 & 3.5 & 56.0 \\
\hline IM route, n (\%) & $3(0.7)$ & $12(3.8)$ & $14(6.1)$ & $29(2.9)$ \\
\hline Median & 37.5 & 20.6 & - & 37.5 \\
\hline UK licensed maxir & & & & \\
\hline
\end{tabular}

Note: Gulf States, represents combined data from Kuwait and the United Arab Emirates. Abbreviation: IM, intramuscular. 
Table 6 Summary of SGA treatment as monotherapy/combination therapy during hospitalization

\begin{tabular}{|c|c|c|c|c|}
\hline & Egypt $(n=480)$ & Saudi Arabia $(n=333)$ & Gulf States $(n=244)$ & Overall $(n=I, 057)$ \\
\hline Patients with at least one SGA medication, $\mathrm{n}(\%)$ & $459(95.6)$ & $318(95.5)$ & $234(95.9)$ & I,0II (95.6) \\
\hline One SGA (monotherapy), n (\%) & $78(16.3)$ & $61(18.3)$ & $4 \mid(16.8)$ & $180(17.0)$ \\
\hline More than SGA only, $\mathrm{n}(\%)$ & $59(12.3)$ & $53(15.9)$ & $27(I I . I)$ & $139(13.2)$ \\
\hline SGA combined with other antipsychotic medications, $\mathrm{n}(\%)$ & $322(67.1)$ & $204(6 \mid .3)$ & $166(68.0)$ & $692(65.5)$ \\
\hline $\mathrm{SGA}+\mathrm{FGA}, \mathrm{n}(\%)$ & $139(29.0)$ & $63(18.9)$ & $18(7.4)$ & $220(20.8)$ \\
\hline SGA + other, $\mathrm{n}(\%)$ & $68(14.2)$ & $87(26.1)$ & $119(48.8)$ & $274(25.9)$ \\
\hline SGA + FGA + other, n (\%) & $115(24.0)$ & $54(16.2)$ & $29(11.9)$ & $198(18.7)$ \\
\hline
\end{tabular}

Notes: Other indicates other psychiatric medication (antiepileptics, antidepressants, anxiolytics, anticholinergic agents, and antidementia drugs). Gulf States, represents combined data from Kuwait and the United Arab Emirates.

Abbreviations: FGA, first-generation antipsychotic; SGA, second-generation antipsychotic.

\section{Maintenance therapy upon discharge}

Upon discharge, the vast majority of study patients ( $\mathrm{n}=992$, 93.9\%) were prescribed SGAs as maintenance therapy (Table 7). Of the 1,011 patients treated with an SGA during hospitalization, 857 (84.8\%) were prescribed the same medication(s) at discharge.

\section{Discussion}

This study provides observational evidence for the duration of hospitalization and type, dose, and mode of administration of SGAs in the management of patients with an acute episode of schizophrenia in the Middle East. There were differences between the treatment recommendations for schizophrenia published by the American Psychiatric Association and observed clinical practice despite guidelines recommending that SGAs should be administered preferably as monotherapy. Only $17 \%$ of individuals received SGA medication as monotherapy in this study, while $65.5 \%$ of patients were treated with polypharmacy following hospitalization. These findings echo an observational study in Europe ${ }^{12}$ demonstrating that combination therapy was commonly used for the treatment of schizophrenia. In this study, over half of the patients were not being treated with antipsychotics in the month prior to hospitalization. This could have arisen if these patients were newly diagnosed with schizophrenia, and could explain why their symptoms became exacerbated.
Some patients' symptoms may also have been better managed by polytherapy, with antipsychotic agents being used to manage psychosis and other medications providing sedation or agitation control. Notably, only $9.3 \%$ of patients were treated with anxiolytics. While this may be surprising because anxiety is commonly associated with an increased risk of relapse and suicide in patients with schizophrenia, and the use of anxiolytics could in turn be expected to be considerably higher, ${ }^{14}$ in this study, patients' agitation tended to be controlled with haloperidol and promethazine.

In this study, the vast majority of antipsychotics were administered orally rather than intramuscularly. Certainly in Egypt this probably occurred because long-acting injectable therapies are more expensive than oral therapies and are not covered by any health insurance program. Additionally, some patients prefer to receive medication daily rather than less frequently (eg, once fortnightly or once monthly). Differences were observed between the choices of antipsychotics administered in the countries in this study. Although some drugs may be recommended in the guidelines, not all were available in the countries included. For example, Egypt tended to use more FGAs in combination therapy than the Gulf States and Saudi Arabia. This may have occurred because FGAs are less costly than SGAs. ${ }^{15}$ The choice of SGAs may also have been affected by hospital formulary decisions, in that certain medications may not have been

Table 7 Summary of antipsychotic treatment as maintenance therapy

\begin{tabular}{lllll}
\hline & Egypt $(\mathbf{n}=\mathbf{4 8 0})$ & Saudi Arabia $(\mathbf{n}=\mathbf{3 3 3})$ & Gulf States $(\mathbf{n}=\mathbf{2 4 4})$ & Overall $(\mathbf{n}=\mathbf{I}, \mathbf{0 5 7})$ \\
\hline $\begin{array}{l}\text { SGA prescribed as maintenance therapy, } \mathrm{n}(\%) \\
\text { 95\% Cla }\end{array}$ & $447(93.1)$ & $316(94.9)$ & $229(93.9)$ & $992(93.9)$ \\
$\begin{array}{l}\text { Same SGA used during hospitalization } \\
\text { and at discharge, }{ }^{\mathrm{b}} \mathrm{n}(\%)\end{array}$ & $(90.5-95.2)$ & $(92.0-97.0)$ & $(90.1-96.5)$ & $(92.2-95.2)$ \\
$\quad$ & & & \\
Yes & $381(83.0)$ & $268(84.3)$ & $208(88.9)$ & $857(84.8)$ \\
No & $78(17.0)$ & $50(15.7)$ & $26(11.1)$ & $154(15.2)$ \\
\hline
\end{tabular}

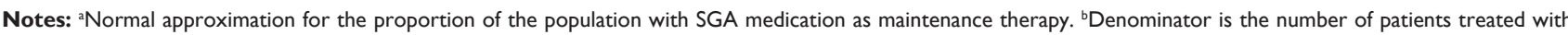
SGA medication during hospitalization. Gulf States, represents combined data from Kuwait and the United Arab Emirates.

Abbreviations: SGA, second-generation antipsychotic; $\mathrm{Cl}$, confidence interval. 
available in all of the hospitals. For example, the availability of antipsychotics may have varied by sector; medications that are available in the private sector may be different to those in the public sector. Furthermore, the antipsychotics available in different hospitals within the same sector may be affected by the demand for them; consequently, patients stable on one antipsychotic may be shifted to another antipsychotic because of limited supplies of a particular antipsychotic agent. In addition, the choice of SGAs administered may have been influenced by both the length of diagnosis of schizophrenia and the patient's comorbidity.

Interestingly, although differences in the doses of SGAs administered were observed, this was not thought to reflect differences in economic circumstances or drug pricing between the countries. The doses of risperidone, olanzapine, and quetiapine were higher in Egypt than in Saudi Arabia or the Gulf States; although clozapine was used more frequently in Egypt, it was also used at lower doses than in the other countries, probably because it was being used as a sedative to reduce agitation. While the doses of SGAs were largely within the guidance specified on the product labeling, the median doses of olanzapine (20.0, 17.5, and $15.0 \mathrm{mg}$ in Egypt, Saudi Arabia, and the Gulf States, respectively) suggest that some patients were receiving more than the recommended maximum dose of $20 \mathrm{mg}$ per day. ${ }^{9}$

The length of hospital stay also varied between countries, with patients having shorter hospital stays in Egypt than in Saudi Arabia and the Gulf States, which could be explained by several possible factors. Due to economic constraints, patients could have been discharged earlier in Egypt than in the other countries to reduce hospital costs. Use of electroconvulsive therapy followed national guidelines, and was notably higher in Egypt than in Saudi Arabia or the Gulf States, where it may have contributed to a reduced length of hospital stay. ${ }^{7,8}$ In Saudi Arabia, patients received significantly less psychological treatment than in Egypt, presumably in part because doctors receive very little psychotherapy training after qualifying and there is a national shortage of clinical psychologists; this could potentially contribute to longer patient stays in Saudi Arabia than in Egypt. In Saudi Arabia and the Gulf States, some families and guardians were reported to be reluctant to take patients out of hospital for social or family reasons. This could lead to extended hospital stays over and above what is necessary for medical reasons, and may also potentially contribute to longer patient stays compared with Egypt. All countries used mainly combination therapy in this study, suggesting that using polypharmacy to reduce the length of hospital stay had an equivalent effect across the study population, and is not a differentiating factor in the variation in length of stay.

In this study, patients were very rarely assessed by rating scales. We postulate that since all patients by definition had severe symptoms leading to hospitalization, physicians felt it unnecessary to determine a specific measurement. Although the patient's medical history should be used to determine the choice of medication according to most guidelines, in this study, the factors most commonly used to select medication were patients' current symptoms in Egypt (80\%) and Saudi Arabia (61\%), and physicians' personal clinical experience in the Gulf States (41.7\%). However, we consider these definitions somewhat overlapping, and hence there is limited significance attached to this disparity. Perhaps surprisingly, patients' co-occurring medical conditions were not cited as a major consideration in choice of medication. The vast majority $(84.8 \%)$ of patients were prescribed the same drug as maintenance therapy at discharge as during hospitalization; a possible explanation for the $15 \%$ of patients who were prescribed a different maintenance therapy was they may have had their combination therapy reverted to monotherapy.

One of the most striking factors in this study was the number of male patients enrolled. The prevalence of schizophrenia is significantly higher in males than in females (male to female ratio $1.4: 1),{ }^{16}$ which could in part explain why there were more male than female participants in the study. The observed sex difference in this study probably also reflects cultural differences in the Middle East compared with other countries, where families are reluctant to admit women to hospital for fear of stigma. This suggests that there are many female patients experiencing relapse/exacerbation of symptoms who are not receiving appropriate care. In Egypt, a greater number of patients reported making suicide attempts in the previous 2 years compared with Saudi Arabia and the Gulf States. A higher level of education has been reported to be a risk factor for later suicide in schizophrenic patients, ${ }^{17}$ and consistently, patients from Egypt in this study had a greater mean number of education years than patients in Saudi Arabia or the Gulf States. Alcohol and illicit drugs may worsen the symptoms of schizophrenia, for example, by causing depression or psychosis or by reducing the effectiveness of medications for schizophrenia. ${ }^{18}$ Since only around $4 \%$ and $14 \%$ of patients reported recent alcohol or illicit drug abuse, respectively, in this study, these risk factors are probably much less important in disease progression in the Middle East than in other countries where the incidence of drug and alcohol abuse is far higher. ${ }^{19}$ 
As with any research, there were certain limitations in this study. For example, although data were collected from a large population of patients in four countries, these may not provide a complete representation of the population of each country. Given that other countries also exist within the Middle East, it is difficult to draw conclusions regarding the complete clinical situation for the treatment of schizophrenia in the whole of the Middle East based on the results of one study. Moreover, with the reluctance of families to admit women to hospital in the Middle East, it is difficult to study accurately how women are treated for acute psychotic episodes within the region.

In conclusion, this study indicates that current clinical practice for the treatment of acute psychotic episodes during hospitalization in the Middle East differs from guidelines published by organizations including the American Psychiatric Association. Although the majority of patients were treated with SGAs, these agents were commonly used in combination with other psychiatric medications, rather than as monotherapy. Most antipsychotics were administered orally, with preferred drugs and average doses varying by country.

\section{Acknowledgments}

Statistical analysis of the study data was carried out by Mark McBride, of Instat Services (Chatham, NJ, USA), supported by AstraZeneca. Editorial assistance was provided by Toby Galbraith and Charlotte Simpson of IMC Healthcare Communication Ltd (London, UK), and was funded by AstraZeneca.

\section{Author contributions}

SA, NAZ, SK, TO, TD, HR, and TMT were national coordinating investigators in the study, were involved in conduct of the study, and collected data. All authors contributed to analysis of the data and critical review of the manuscript, and approved the final manuscript.

\section{Disclosure}

This study was funded by AstraZeneca. The study protocol was designed in close collaboration between regional and local AstraZeneca offices to address the aim of the study of identifying gaps in the available data in targeted countries. The draft protocol was reviewed by local principal investigators and steering committees and amended as necessary. The final results and report of the study were reviewed and amended by the principal and national coordinators, and approved by them prior to finalization.
AstraZeneca did not influence analysis of the data, which was conducted centrally by a service provider. HR, SA, and NAZ received honoraria from AstraZeneca. HR has also received honoraria from Pfizer, Lilly, Abbott, Lundbeck, Sanofi Aventis, and Johnson \& Johnson. The other authors report no conflicts of interest in this work.

\section{References}

1. van Os J, Kapur S. Schizophrenia. Lancet. 2009;374(9690):635-645.

2. World Health Organization. Mental health: schizophrenia; 2012. Available from: http://www.who.int/mental_health/management/ schizophrenia/en/. Accessed February 19, 2015.

3. Ayuso-Mateos JL. Global burden of schizophrenia in the year 2000 version 1 estimates. Available from: http://www.who.int/healthinfo/ statistics/bod_schizophrenia.pdf. Accessed February 19, 2015.

4. World Health Organization. Age-standardized DALYs per 100,000 by cause and Member State; 2004. Available from: www.who.int/ healthinfo/global.../gbddeathdalycountryestimates2004.xls. Accessed February 19, 2015.

5. Gorwood P. Meeting everyday challenges: antipsychotic therapy in the real world. Eur Neuropsychopharmacol. 2006;16 Suppl 3: S156-S162.

6. Valenstein M, Ganoczy D, McCarthy JF, Myra KH, Lee TA, Blow FC. Antipsychotic adherence over time among patients receiving treatment for schizophrenia: a retrospective review. J Clin Psychiatry. 2006; 67(10):1542-1550.

7. Okasha T. The practice of electroconvulsive therapy (ECT) in a sample of Egyptian patients. Curr Psychiatr. 2006;13(1):105-114.

8. Okasha T. Electro-convulsive therapy (ECT): an Egyptian perspective. Afr J Psychiatry. 2007;10:22-24.

9. American Psychiatric Association. Practice Guideline For the Treatment of Patients With Schizophrenia, Second Edition. Available from: http:// psychiatryonline.org/pb/assets/raw/sitewide/practice_guidelines/guidelines/schizophrenia.pdf. Accessed February 19, 2015.

10. Taylor D, Paton C, Kapur S. Prescribing Guidelines in Psychiatry. 11th ed. Oxford, UK: Wiley-Blackwell; 2012.

11. American Psychiatric Association. Practice guidelines for the treatment of patients with schizophrenia. 2nd ed. Am J Psychiatry. 2004; $161: 1-56$

12. Barnes TR. Evidence-based guidelines for the pharmacological treatment of schizophrenia: recommendations from the British Association for Psychopharmacology. J Psychopharmacol. 2011;25(5):567-620.

13. Haro JM, Novick D, Belger M, Jones PB. Antipsychotic type and correlates of antipsychotic treatment discontinuation in the outpatient treatment of schizophrenia. Eur Psychiatry. 2006;21(1):41-47.

14. Luchins D. Typical versus atypical antipsychotics. Am J Psychiatry. 2004;161(10):1927.

15. Garay RP, Samalin L, Hameg A, Llorca PM. Investigational drugs for anxiety in patients with schizophrenia. Expert Opin Investig Drugs. 2014;25:1-11.

16. McGrath J, Saha S, Chant D, Welham J. Schizophrenia: a concise overview of incidence, prevalence and mortality. Epidemiol Rev. 2006; 30:67-76.

17. Hor K, Taylor M. Suicide and schizophrenia: a systematic review of rates and risk factors. J Psychopharmacol. 2010;24 Suppl 4: 81-90.

18. Lehman AF, Lieberman JA, Dixon LB, et al. Practice Guideline for the Treatment of Patients with Schizophrenia, Second Edition, 2004. Available from: http://psychiatryonline.org/pdfaccess.ashx?Resource $\mathrm{ID}=243185 \&$ PDFSource=6. Accessed February 19, 2015.

19. World Health Organization. Global status report on alcohol and health, 2005-2011. Available from: http://www.who.int/substance_abuse/ publications/global_alcohol_report/msbgsruprofiles.pdf. Accessed February 19, 2015. 


\section{Supplementary material}

Table SI Principal investigators in this study

\begin{tabular}{|c|c|c|}
\hline Name & City & Country \\
\hline Marwa Abdel Megeed Hamed & Cairo & Egypt \\
\hline Yaser Abd El Qawy Salem & Alexandria & Egypt \\
\hline Wael Abohendy & Zagazig & Egypt \\
\hline Samir Mohamed Fouad Abo El Magd & Cairo & Egypt \\
\hline Raed Al Ghamdi & Riyadh & Saudi Arabia \\
\hline Abdullah AI Hathloul & Riyadh & Saudi Arabia \\
\hline Sulaiman Al Khadhari & Kuwait & Gulf States \\
\hline Nasir Al Zein & Dammam & Saudi Arabia \\
\hline Lotfy Abd El Aziz El Sherbiny & Alexandria & Egypt \\
\hline Mohamed Hashem Kamal Hashem Bahary & Cairo & Egypt \\
\hline Bahjat Balbous & Dubai/United Arab Emirates & Gulf States \\
\hline Tarek Abdullah Darwish Selim & Abu Dhabi/United Arab Emirates & Gulf States \\
\hline Tamer Abd El Rahman El Amrousy & Cairo & Egypt \\
\hline Sohir Helmy Ibrahim El Ghoneimy & Cairo & Egypt \\
\hline Amgad Ahmed Abdel Ghani El Agroudi & Cairo & Egypt \\
\hline Adel Elashashay & Alexandria & Egypt \\
\hline Ahmed Mohamed Refaat Eldesouky & Helwan - Cairo & Egypt \\
\hline Ayman Elhaddad & Abha & Saudi Arabia \\
\hline Adel Mohamed El Medany & Cairo & Egypt \\
\hline Nahla Elsayed Nagi & Cairo & Egypt \\
\hline Mahmoud Ahmed Awad El Wassify & Mansoura & Egypt \\
\hline Magda Taha Kamal El Din Fahmy & Cairo & Egypt \\
\hline Mohamed Farid AboElhoda & Talkha - Aldakahlia & Egypt \\
\hline Hossam El-Din Fathallah El Sawy & Tanta & Egypt \\
\hline Atef Ali Ahmed Fayed & Alexandria & Egypt \\
\hline Mohamed Ghazi & Dammam & Saudi Arabia \\
\hline Hany Hamed Eldessoky & Cairo & Egypt \\
\hline Ai-Amin Hani & Jeddah & Saudi Arabia \\
\hline Khaled Moustafa Ibrahim Helmy & Alexandria & Egypt \\
\hline Khaled Shera & Jeddah & Saudi Arabia \\
\hline Suhail Khan & Jeddah & Saudi Arabia \\
\hline Riadh Khoudir & Ras Al-Khaimah/United Arab Emirates & Gulf States \\
\hline Talaat Matar Tadros & Ras Al-Khaimah/United Arab Emirates & Gulf States \\
\hline Mohamed Elbahy & Shebin El-Kom & Egypt \\
\hline Tarek Kamal Molokheia & Alexandria & Egypt \\
\hline Sherif Nazeer Aziz Meseeha & Cairo & Egypt \\
\hline Ahmed Refaat Ibrahim Daebes & Alexandria & Egypt \\
\hline Khaled Mohamed Sabry El Attar & Cairo & Egypt \\
\hline Ashraf Said Hanafy & Cairo & Egypt \\
\hline Ola Omar Hassan Shaheen & Cairo & Egypt \\
\hline Ehab Shawky Mohamed Metwally & Tanta & Egypt \\
\hline Youssef Shawosh & Al Taif & Saudi Arabia \\
\hline Asharaf Tantawi & Buridah & Saudi Arabia \\
\hline Adel Youssef Abdel Meseeh & Cairo & Egypt \\
\hline Magda Zaki & Dubai/United Arab Emirates & Gulf States \\
\hline
\end{tabular}

Neuropsychiatric Disease and Treatment

\section{Publish your work in this journal}

Neuropsychiatric Disease and Treatment is an international, peerreviewed journal of clinical therapeutics and pharmacology focusing on concise rapid reporting of clinical or pre-clinical studies on a range of neuropsychiatric and neurological disorders. This journal is indexed on PubMed Central, the 'PsycINFO' database and CAS,

\section{Dovepress}

and is the official journal of The International Neuropsychiatric Association (INA). The manuscript management system is completely online and includes a very quick and fair peer-review system, which is all easy to use. Visit http://www.dovepress.com/testimonials.php to read real quotes from published authors. 